\title{
On the efficiency and correction of vertically oriented blunt bioaerosol samplers in moving air
}

\author{
Dominik Michel • Mathias W. Rotach • Regula Gehrig • \\ Roland Vogt
}

Received: 9 May 2011 / Revised: 30 January 2012 / Accepted: 30 January 2012

(C) ISB 2012

\begin{abstract}
The aspiration efficiency of vertical and windoriented Air-O-Cell samplers was investigated in a field study using the pollen of hazel, sweet chestnut and birch. Collected pollen numbers were compared to measurements of a Hirst-type Burkard spore trap. The discrepancy between pollen counts is substantial in the case of vertical orientation. The results indicate a strong influence of wind velocity and inlet orientation relative to the freestream on the aspiration efficiency. Various studies reported on inertial effects on aerosol motion as function of wind velocity. The measurements were compared to a physically based model for the limited case of vertical blunt samplers. Additionally, a simple linear model based on pollen counts and wind velocity was developed. Both correction models notably reduce the error of vertically oriented samplers, whereas only the physically based model can be used on independent datasets. The study also addressed the precision error of the instruments used, which was substantial for both sampler types.
\end{abstract}

Keywords Burkard sampler · Air-O-Cell sampler - Orifice orientation $\cdot$ Birch pollen $\cdot$ Field measurement

D. Michel $(\bowtie) \cdot R$. Vogt

Meteorology, Climatology and Remote Sensing,

University of Basel,

Basel, Switzerland

e-mail: dominik.michel@unibas.ch

D. Michel $\cdot$ M. W. Rotach $\cdot$ R. Gehrig

Federal Office of Meteorology and Climatology MeteoSwiss,

Zurich, Switzerland

M. W. Rotach

Institute of Meteorology and Geophysics, University of Innsbruck,

Innsbruck, Austria

\section{Introduction}

Experimental investigation of local aerosol dispersion requires both spatially and temporally detailed information on their concentrations. Spatial resolution is often limited by the availability of instruments. Therefore, different instrument types may be used in order to increase the instrumental set-up, i.e., spatial information. The comparability of different aerosol sensors, however, presumes a knowledge of their aspiration characteristics. The present study was motivated by a field experiment (Michel et al. 2010) in which different bioaerosol samplers were used to sample birch pollen (22 $\mu \mathrm{m}$ diameter). The measurements indicate a substantial discrepancy between wind-oriented Hirst-type (Hirst 1952) Burkard samplers and vertically oriented Air-O-Cell samplers. The latter were facing upwards to make the measurements independent of wind direction. In the present study, field intercomparison of Burkard and Air-O-Cell samplers was carried out during the birch pollen season in 2010. The Air-O-Cells were oriented vertically as well as horizontally. The effect of orifice orientation on the aspiration efficiency (i.e., the ratio of sampled aerosols to the aerosol number in the considered volume of air upstream of the orifice) is wellknown (Ogden et al. 1974). Aspiration is related to inertial effects on particle motion. As the angle between the orifice and the freestream increases, the behavior of the flow approaching a sampler is progressively complicated, and the aspiration efficiency decreases dramatically (Lundgren et al. 1978; Vincent et al. 1986). Thus, in the case of vertically oriented blunt samplers, which represents most pollen samplers used in the field (Levetin 2004), a correction of the measured data is indispensable. It would be of great benefit to bioaerosol-related research (e.g., the advancing field of aeroallergenic studies), if corrections of measured data were performed at experimental as well as 
operational stations. The impaction efficiency of Air-O-Cell and Burkard samplers (i.e., the ratio of sampled aerosols to the number of aspirated aerosols) was investigated under laboratory conditions by Aizenberg et al. (2000). Their results indicate a good agreement of the Air-O-Cell with the Burkard sampler for small fungal and bacterial aerosols $(<3.5 \mu \mathrm{m})$. The aspiration efficiency of the Burkard sampler was determined under laboratory conditions with respect to wind velocity and pollen size by Hirst (1952) and Ogden et al. (1974). For Ambrosia pollen, which have a similar size as birch pollen $(20 \mu \mathrm{m})$, Ogden et al. (1974) found an efficiency declining to $60 \%$ for wind velocities $<5 \mathrm{~ms}^{-1}$. A physically based semi-empirical model accounting for the effects of vertical oriented sampler inlets, wind velocity and bluntness of the sampler body on aerosol motion has been developed by Tsai and Vincent (1993). In the present work, this aspiration model was verified with the measured data of the sampler intercomparison. The deviation of vertical AirO-Cells from Burkard measurements could be reduced notably. Additionally, an alternative linear correction model was constructed on the basis of pollen counts and wind velocity. The linear correction yields a better agreement of Air-O-Cells with the Burkard sampler, yet it cannot be transferred to independent datasets.

Apart from aspiration efficiency, uncertainties are also induced by the manual sample analysis, which is still the standard at most operational pollen observation sites. Large errors may occur when aerosol deposition on the impaction area is inhomogeneous. In the present study, this applies only to the Burkard sampler, since the Air-O-Cell counting covers the entire impaction area. Laboratory and field studies (Comtois et al. 1999; Aizenberg et al. 2000; Gottardini et al. 2009) on pollen density distribution on the slide of volumetric pollen samplers showed that the distribution is mostly inhomogeneous and dependent on pollen size.

\section{Materials and Methods}

Pollen sampling methods

The instrument set-up included two different types of bioaerosol samplers, which both use the impaction principle, where aerosols impinge on a sampling substrate as an effect of greater inertia than that of air, accelerated by aspiration. The Air-O-Cell (A) sampler (Zefon International, http:// www.zefon.com; Levetin 2004) is a disposable cylindrical cassette, which includes a sampling substrate-coated glass slide. Its orifice tapers to a slit of $14.4 \mathrm{~mm} \times 1 \mathrm{~mm}$. The instrument can be mounted wind-oriented (horizontally) when the wind direction is persistent, or facing upwards (vertically), making the measurements independent of wind direction. In the present study both orientations were used.
The Air-O-Cell cassette needs to be connected to a pump, which is capable of producing an sampling rate of $15 \mathrm{lmin}^{-1}$. The pump provided by Zefon is not suitable for experimental outdoor use and very costly if a larger array of A samplers is to be equipped. Therefore, an alternative selfconstructed aspiration system consisting of parallel membrane pumps was attached to the cassettes conferring a cylindrical shape on the total A sampler (see Fig. 2). The system provides a sampling rate of $18.51 \mathrm{~min}^{-1}$, which exceeds the factory-recommended minimal sampling rate. A larger sampling rate increases the range of sampled particles towards lighter particles. The temporal resolution is determined by the cassette exposure. The sampling rate was monitored in situ using a thermal gasflowmeter (red-y smart, Vögtlin Instruments, Aesch, Switzerland). The exposure time was $45 \mathrm{~min}$, then the cassettes were replaced. The collected pollen numbers were extrapolated to obtain a 1-h resolution. The pollen counting procedure covered the entire impaction area.

The Burkard (B) sampler (Burkard Manufacturing, Rickmansworth, UK) is a wind-oriented instrument with a $2 \mathrm{~mm} \times 14 \mathrm{~mm}$ slit orifice (Mandrioli et al. 1998). The sampling rate is $101 \mathrm{~min}^{-1}$. Particles are collected on an adhesive sampling tape mounted on a rotating cylinder, which provides a continuous 7-day information on pollen concentration. The temporal resolution depends on the analysis method. In the present study, a 1-h resolution was obtained. Four or five longitudinal counting sweeps were performed across the impaction area, which yields a coverage of 20.5 and $25.7 \%$, respectively. The total pollen number was extrapolated to the entire impaction area. The body shape (i.e., bluntness) is similar to that of the human head, since the B sampler was designed to measure inhalable aerosols rather than their atmospheric concentration. The B sampler is used commonly in European bioaerosol monitoring networks. In the present study, therefore, their measurements are taken as a reference.

Sampler intercomparison experiment

The sampler intercomparison was conducted in Illarsaz, Switzerland during the emission period of birch (Betula) pollen from 5-23 April 2010. The study site (Fig. 1) was located in a valley where birch is common. The region is dominated by a plains-mountain wind system with persistent wind conditions (mean wind direction $350^{\circ}$ during daytime and southerly wind during the night). Knowledge of the predominant daytime wind direction was used to align the instrument set-up normal to the mean wind direction. A small spatial instrument separation $(<10 \mathrm{~m})$ was chosen to minimize sampling discrepancies due to inhomogeneities of the atmospheric pollen distribution (Fig. 2). In the case of 
Fig. 1 Satellite images of the experiment locations in Illarsaz, Switzerland (top left Lake of Geneva). Inset, lower left Location of intercomparison experiment 2010 (a). Inset, top right The frequency of wind direction and velocity measured at $a$. Federal Office of Topography swisstopo (http://www.swisstopo.admin. $\mathrm{ch} /$ internet/swisstopo/en/home. html)

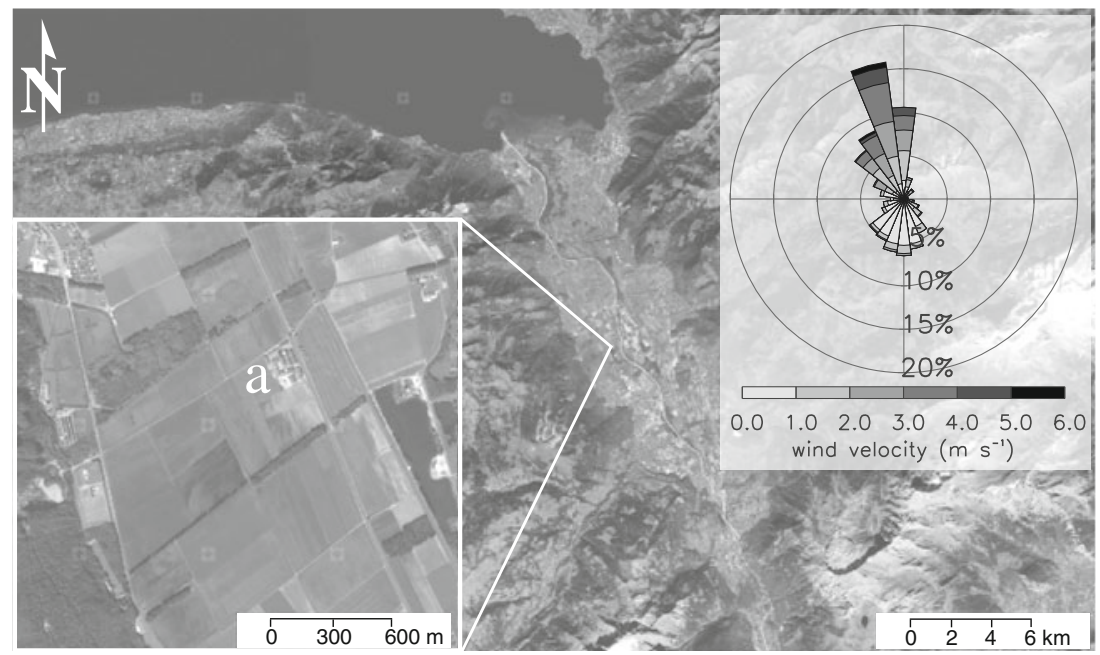

low concentrations, however, atmospheric inhomogeneities may induce large relative sampling errors of 1-h averages. The measuring height was $2 \mathrm{~m}$ above the ground.

The intercomparison set-up consisted of two B samplers and four A samplers; two A samplers were oriented horizontally $\left(A_{h}\right.$, where the subscript refers to orientation), facing the mean wind. Two A samplers were oriented vertically $\left(A_{v}\right)$, facing upwards. This set-up was chosen in order to assess the difference between the $\mathrm{A}$ and $\mathrm{B}$ sampler when both are mounted such that the orifice faces the mean wind direction, as well as the impact of vertical orientation of the A sampler. Simultaneously, a USA-1 ultrasonic anemometer (METEK, Elmshor, Germany, $10 \mathrm{~Hz}$ ) was mounted at $2 \mathrm{~m}$ above the ground and $21 \mathrm{~m}$ upwind of the bioaerosol samplers to monitor the wind velocity. The B samplers as well as the sonic anemometer were operated during the entire campaign period. The A samplers were operated only during periods of favourable wind conditions (i.e., northerly wind direction).

Additional data were available for different pollen species [hazel (Corylus), $28 \mu \mathrm{m}$ average diameter and sweet chestnut (Castanea), $14 \mu \mathrm{m}$ average diameter, Bucher et al. (2004)]. These shorter intercomparisons were conducted in 2009 using a similar experimental arrangement as in Fig. 2, but at operational MeteoSwiss pollen monitoring sites.

\section{Post-processing}

The pollen concentration of B samples was calculated according to the recommendations of Mandrioli et al. (1998). In the case of A samplers, the pollen concentration was calculated as the ratio of the total pollen count and the aspirated volume of air. Since the exposure time was only $45 \mathrm{~min}$, the concentration was extrapolated to $1 \mathrm{~h}$. This may have led to a certain error when compared to B measurements, because pollen transport occurs as intermittent gusts rather than a steady pollen flux.

With respect to the azimuth of the horizontal A samplers $\left(350^{\circ}\right)$, only wind directions related to the mean wind direction $\pm 50^{\circ}$ were accepted for analysis.
Fig. 2 Set-up of instruments in the sampler intercomparison in Illarsaz 2010. Sampler $B-I$ was mounted at the far right side of this figure. The coordinate system denotes the orientation of the wind components relative to the instrument orientation. Measurements from the Burkard Scientific sampler $(B-I I I)$ were not used in the present study. The dimensions of the A sampler including the pump housing are specified for $A v-I$

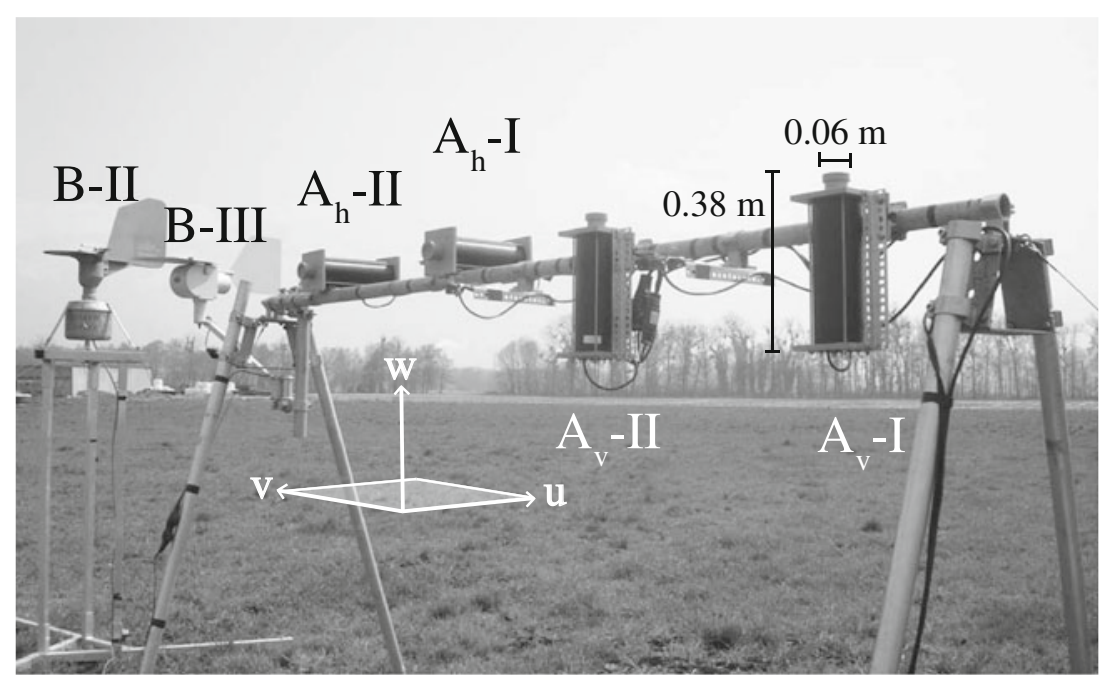


Overview on the sampler agreement

Prior to the comparison of different sampler types, the precision of each sampler type $B, A_{V}$ and $A_{h}$ was addressed separately on the basis of sweet chestnut and birch pollen measurements by analyzing pairs of identical sampler types.

The dataset of hazel pollen was not taken into account because the sample size was too small. The measurements indicate a good correlation of all sampler types $\left(R^{2} \geq 0.61\right.$, Table 1). The root mean square error (rmse) is used to quantify the precision of the instruments. The relative rmse is calculated as rmse normalized by $\langle\bar{c}\rangle$, where $\langle\bar{c}\rangle$ is the spatial and temporal average of the two measurements (e.g., $\left.A_{v}-I / I I\right)$. The relative rmse of the $A$ and $B$ samplers is rather large (from $26 \%$ to $57 \%$ ). The large error among the vertically oriented A samplers may have resulted from strong statistical effects of atmospheric inhomogeneities in the case of low $\langle\bar{c}\rangle$ measurements. In contrast, horizontal A samplers agree comparably well, since the observed $\langle\bar{c}\rangle$ is considerably larger. The large uncertainty among the B samplers arises from the counting procedure rather than from the aspiration efficiency. The agreement among identical sampler types is summarized in Table 1 .

A re-analysis of three $B$ slides was performed in order to quantify the influence of the number of sweeps on the calculated pollen concentration. The slides have been recounted on the basis of 24-h -slides with 4 and 30 longitudinal sweeps of $0.36 \mathrm{~mm}$ width, which yields a coverage of $10 \%$ and $77 \%$, respectively, of the impaction area $(48 \times$ $14 \mathrm{~mm}$ ). Figure 3 illustrates that the position of the sweeps has a strong influence on the extrapolated total, if the distribution is not homogeneous (e.g., bipolar). Cariñanos et al. (2000) investigated different pollen counting methods of slides using a Hirst-type sampler. They found an overestimation of extrapolated pollen numbers when the sweeps are positioned in the middle of the slide, where most pollen are deposited. The present counting method considered depositions near the edge of the impaction area as well in the center. The variation in the numbers of sweeps yielded a relative error of $16 \%$, when only $10 \%$ of the deposition area

Table 1 Precision of different sampler types. The relative root mean square error (rmse) is calculated as rmse normalized by $\langle\bar{c}\rangle$, where rmse denotes the root mean square difference of two samplers (I and II) with the same orientation and $\langle\bar{c}\rangle$ is the spatial and temporal average of the two samplers. $n$ Sample size

\begin{tabular}{lllll}
\hline Pollen species sampler & $\begin{array}{l}\text { Sweet chestnut } \\
\text { Av-I/II }\end{array}$ & $\begin{array}{l}\text { Birch } \\
\text { Av-I/II }\end{array}$ & $\begin{array}{l}\text { Birch } \\
\text { Ah-I/II }\end{array}$ & $\begin{array}{l}\text { Birch } \\
\text { B-I/II }\end{array}$ \\
\hline$R^{2}$ & 0.61 & 0.89 & 0.96 & 0.90 \\
relative rmse [\%] & 37 & 57 & 26 & 38 \\
$\langle\bar{c}\rangle\left[\right.$ pollen m $\left.{ }^{-3}\right]$ & 19.0 & 25.3 & 684.7 & 848.9 \\
$n$ & 24 & 47 & 47 & 47 \\
\hline
\end{tabular}

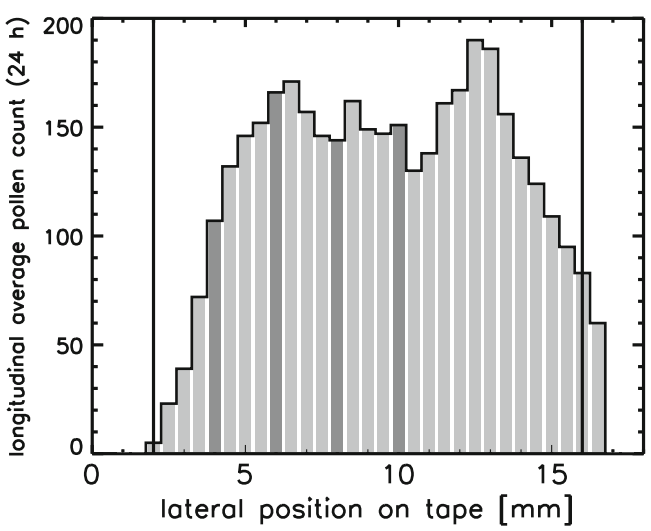

Fig. 3 Lateral pollen deposition on the Burkard sampling slide obtained from 24-h averages of longitudinal pollen counts. Gray area Absolute pollen counts ( $y$-axis) and analyzed area ( $x$-axis) when 30 sweeps were performed, dark gray bars four sweeps only performed, black vertical lines outer limits of impaction area, which corresponds to the inner inlet orifice $(2 \times 14 \mathrm{~mm})$

was analysed instead of 77\%. Comtois et al. (1999) found a larger relative error (almost 30\%) in the case of four sweeps (slide coverage of $13.3 \%$ ), when $100 \%$ of the entire slide was analyzed.

\section{Quantification of the error induced by vertical orientation}

For the following sampler intercomparison, spatial averages of aspirated pollen concentrations, $c$, i.e., for example $\left\langle c_{A v}\right\rangle$, are analyzed, denoted as $c_{A v}, c_{A h}$ and $c_{B}$, respectively, where the subscripts refer to sampler type and orientation. The bias denotes the mean deviation of a data series from the reference. The relative bias is calculated as bias normalized by $\bar{c}$ of the reference instrument. The relative rmse is calculated as rmse normalized by $\bar{c}$ of the reference instrument.

Figure 4 illustrates that the aspiration efficiency is decreased when the sampler orifice is facing upwards. Hence, the vertically oriented samplers underestimated the pollen concentration dramatically in comparison to the B sampler. In the case of sweet chestnut pollen, the relative bias was $-70 \%$. The birch pollen measurements underestimated the concentration with an error of almost three orders of magnitude, with a relative bias of $-104 \%$. The better agreement of the sweet chestnut measurements is a result of their lower inertia compared to the larger birch pollen. The majority of the vertically measured concentrations did not exceed 60 pollen $\mathrm{m}^{-3}$, while $c_{\mathrm{B}}$ observations range up to $5 \times 10^{3}$ pollen $\mathrm{m}^{-3}$

The aspiration efficiency of the horizontal A samplers facing the freestream, in contrast, is considerably better. The $c_{A h}$ data agree well with the $\mathrm{B}$ samplers $\left(R^{2}=0.76\right)$. However, the $A_{h}$ samplers also slightly underestimate the pollen concentration, with a relative bias of $-20 \%$. The impaction efficiency of the A cassette already tends towards $100 \%$ with aerosols of an aerodynamic diameter up to $5 \mu \mathrm{m}$ 


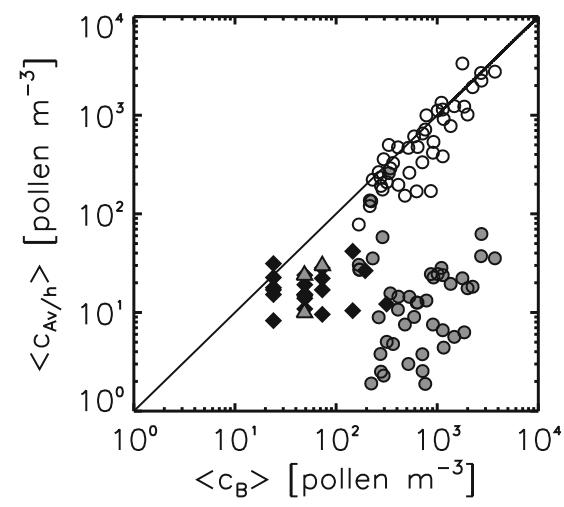

Fig. 4 Agreement of 1-h pollen concentrations measured with B and A samplers; triangles hazel, squares sweet chestnut, circles birch pollen. Filled symbols $\mathrm{A}_{\mathrm{v}}$ data, open symbols $\mathrm{A}_{\mathrm{h}}$ data. In the case of the birch pollen dataset, only cases where the wind direction was $\pm 50^{\circ}$ compared to the azimuth of the $A_{h}$ samplers are shown. Note that the plot is double-logarithmic

(Aizenberg et al. 2000), i.e., much smaller and lighter than birch pollen (22 $\mu \mathrm{m}$ diameter; Birks 1968). Hence, the error of the $A_{h}$ sampler is due mainly to its blunt design, diverting the approaching freestream. The relative bias between birch $c_{A v}$ and $c_{A h}$ amounts to $-105 \%$. This denotes a very large error between identical samplers, which is induced by their orientation. The relative errors of the $A_{v}$ sampler are summarized in Table 3.

Aspiration characteristics of blunt samplers at different orientations

Experimental and theoretical work on the determination of the aspiration efficiency of aerosol samplers related mostly to thin-walled, tubular inlets facing the freestream, e.g., Badzioch (1959) and Belyaev and Levin (1974). This idealization was a starting point to understanding the basic principles that determine aspiration efficiency. In practice, however, the flow pattern upstream of a sampler orifice is much more complex. It is affected by various parameters, such as the orientation, $\theta$, of the orifice relative to the freestream, the aspiration velocity and the characteristic bluntness of the sampler body. Vincent et al. (1986) have investigated the physics of thin-walled samplers with respect to their orientation relative to the freestream. Based on his work, Tsai and Vincent (1993) developed a model for the range of blunt samplers, which was assessed in the present for the single case of vertical orientation. by:

In theory, the aspiration efficiency of a sampler is defined

$\mathrm{E}_{\mathrm{t}}=\mathrm{c} / \mathrm{c}_{0}$,

where $c$ is the particle concentration entering the plane of the sampler inlet, and $c_{0}$ is the upstream concentration, given that the air and particle velocity distributions are uniform. Taking into consideration the influence of inertial effects on the aspiration efficiency due to divergence and convergence of the flow as it approaches the sampler inlet, Eq. 1 can be extended to:

$E=\frac{c}{c_{0}}=1+\beta(R-1)$

where $\beta$ is the impaction efficiency, defined as the ratio between the number of particles impacting onto the virtual plane between the limiting trajectories, and the number of particles impacting onto the plane of the sampling orifice. A robust empirical expression for $\beta$ (Vincent 2007) is:

$\beta=1-\frac{1}{1+G S t}$

where $S t$ is the particle Stokes number and $G$ is an empirical coefficient, which was investigated by Belyaev and Levin (1974) and Paik and Vincent (2002). $R$ is defined as:

$R=\frac{U}{U_{s}}$

where $U$ is the freestream velocity and $U_{\mathrm{s}}$ is the sampling velocity through the sampling plane. The sampling velocity of the A sampler is $U_{\mathrm{s}}=1.84 \mathrm{~ms}^{-1}$. In the case of idealized thin-walled samplers, $R=1$ denotes isokinetic sampling, where $E_{\mathrm{t}}=1$. If sampling is sub-isokinetic $(R>1)$ or superisokinetic $(R<1)$, the trajectories of the air flow are divergent or convergent, respectively, and particles are either gained or lost at the sampler inlet due to inertial effects on the particle motion. With respect to orientations up to $\theta=$ $90^{\circ}$, Eq. 2 can be expressed by an impaction model, which in similar form was used by Belyaev and Levin (1974); Durham and Lundgren (1980); Vincent et al. (1986); Wiener et al. (1988); Lipatov et al. (1988); Vincent (1989); Grinshpun and Lipatov (1990); Hangal and Willeke (1990a, b); Grinshpun et al. (1994) and Vincent (2007):

$E_{\theta}=\frac{c}{c_{0}}=1+\beta_{\theta}(R \cos \theta-1)$

The aspiration performance of a blunt sampler facing upwards in calm air, i.e., for super-isokinetic sampling, has been investigated by Dunnett et al. (2006). The particle Stokes number $S t$ accounts for turbulent particle motion, i.e., the inertially dominated behavior of particles in a distorted flow and is defined as:

$S t=\frac{\tau U}{\delta}$,

where $\delta$ is the sampling orifice width, and $\tau$ is the response time of the particle to perturbation. It can be expressed as:

$\tau=\frac{\gamma^{*} d_{\alpha e}^{2}}{18 \mu}$ 
where $\mu$ is the dynamic molecular viscosity of air, and $d_{\mathrm{ae}}$ is the aerodynamic particle diameter. $d_{\mathrm{ae}}$ characterizes a sphere with the density equivalent to water $\gamma^{*}=1 \mathrm{~g} \mathrm{~cm}^{-3}$ and, therefore, has the same settling velocity as the particle in question. For laminar flow $d_{\mathrm{ae}}$ is defined as:

$d_{\alpha e}=d\left(\frac{\gamma}{\gamma *}\right)^{1 / 2}$,

where $d$ is the particle diameter.

Thus, the Stokes number denotes the ratio of the stopping distance $\tau U_{0}$ of the particle to the width of the sampler orifice, when the particle motion is turbulent, i.e., within distorted flow. Small values of $S t$ indicate that the particles tend to follow the fluid trajectories, while large values indicate a separation of particle motion from the fluid motion.

Based on experimental studies on the human head (Ogden and Birkett 1977; Armbruster and Breuer 1982) and cylindrical thin-walled samplers (Vincent et al. 1986), an aspiration model for the limited case of blunt samplers with orientation $\theta=90^{\circ}$ was developed (Tsai and Vincent 1993). It is expressed as:

$E_{90}=\frac{1}{1+4 G_{90} r^{g 1} S t R^{1 / 2}}$,

where $G_{90}$ and $g_{1}$ are both empirical coefficients. Tsai and Vincent (1993) obtained an agreement of the corrected data of $R^{2}=0.61$ for $G_{90}=2.21$ and $g_{1}=-0.5$, where $G_{90}$ is a scale of the sampler bluntness and $g_{1}$ is a modification of the inlet dimension ratio $r=\delta / D$, where $D$ is the characteristic sampler width. It is important to understand that the area projected upstream by the sampler body, i.e., its bluntness, increases with $\theta$ (Lundgren et al. 1978). Assuming $r=1$, the term $G_{90} r^{\mathrm{g} 1}$ approaches the bluntness of a thin-walled sampler, which was empirically determined to be $G_{\theta}=2.1$ for $\theta$ up to $90^{\circ}$ (Vincent et al. 1986).

An alternative linear correction for vertical aspiration using wind velocity

Aspiration efficiency is dependent strongly on the sampler design, i.e., affected by the characteristic bluntness of the sampler body and the inlet design, described with $\mathrm{r}$. It is unknown to what extent the findings of human head studies (Ogden and Birkett 1977; Armbruster and Breuer 1982; Vincent et al. 1986) apply to the bulky body of the A sampler in the present work. Therefore, an alternative approach to the correction of vertical aspiration is presented, which applies only to the exact experiment sites of the present study and that of Michel et al. (2010).

Based on the knowledge of inertial effects on suspended particle motion, the relation between wind velocity and the discrepancy between $A_{v}$ and $A_{h}$ samplers was determined using the data collected during sampler intercomparison in order to obtain a relative correction of $A_{v}$ against $A_{h}$. The correlation of the corrected $A_{v}$ with the $B$ data was then determined.

Since the $A_{h}$ sampler azimuth is fixed, i.e., not automatically wind-oriented, the effect of yaw orientations needed to be minimized. Therefore, only the wind component $u$ facing the $A_{h}$ sampler orifices with a threshold of $\pm 50^{\circ}$ were accepted for analysis. Hence, $R$ is expressed as $R=\bar{u} / U_{\mathrm{s}}$. Figure 5 shows that the slope of $c_{A v}$ deviations from $c_{A h}$ is steeper, when $R>1$, than for cases where $R<1$. Therefore, the linear regression was calculated separately for cases where $R \leq 1$ and $R>1$. Consequently, the correction procedure incorporates sub-models separated according to the longitudinal wind velocity. The correlation of $\mathrm{A}_{v}$ with $\mathrm{A}_{h}$ data provided two models, which use different sets of coefficients according to values of $\mathrm{u}$ (Eqs. 10, 11):

$c_{\text {lin }}^{*}=\alpha_{1} c_{A v}+\alpha_{2} \bar{u}, \quad$ if $R \leq 1$ and $c_{A v}>0$

$c_{l i n}^{*}=\alpha_{3} c_{A v}+\alpha_{4} \bar{u}, \quad$ if $R>1$ and $c_{A v}>0$,

where $c_{\text {lin }}^{*}$ denotes the corrected pollen concentration.

In a second step, the $c_{\text {lin }}^{*}$ data are fitted against the $\mathrm{B}$ data to decrease the offset indicated in Fig. 4 between $B$ and $A_{h}$ measurements. The resulting linear correction model is defined as:

$c_{\text {lin }}^{* *}=\beta_{1} c_{\text {lin }}^{*}, \quad$ if $c_{\text {lin }}^{*}>0$

where $c_{\text {lin }}^{* *}$ refers to the concentration of the vertically oriented A samplers after the 'double correction'. The goodness-of-fit and coefficients of linear models $c_{\text {lin }}^{*}$ and $c_{\text {lin }}^{* *}$ are shown in Table 2.

\section{Results}

Performance of the physically based aspiration model

The aspiration model for upwards-facing blunt samplers (Eq. 9) was tested against the measured aspiration efficiency

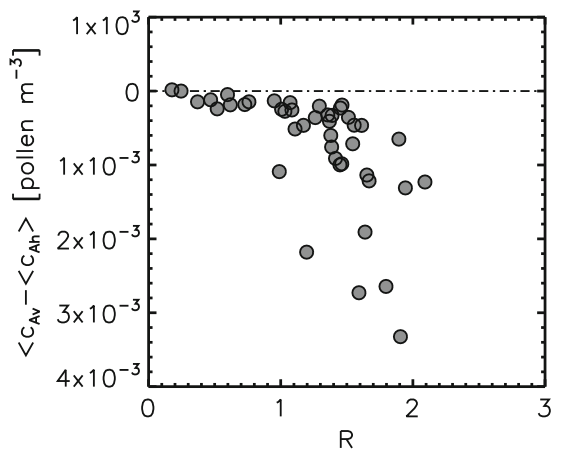

Fig. 5 The difference $c_{A v}-c_{A h}$ as a function of $R=\bar{u} / U_{\mathrm{s}}$, where $\bar{u}$ is the longitudinal wind component, and $U_{\mathrm{s}}$ is the sampling velocity of the Air-O-Cell sampler $\left(1.84 \mathrm{~ms}^{-1}\right)$ 
Table 2 Goodness-of-fit and coefficients of statistical models. $c_{l i n}^{*}$ denotes the linear correction of $\mathrm{A}_{\mathrm{v}}$ data using Eqs. 10 or 11 according to the longitudinal wind velocity component $\overline{\mathrm{u}}, c_{\text {lin }}^{* *}$ denotes the linear correction of $\mathrm{A}_{\mathrm{v}}$ data using Eq. 12

\begin{tabular}{lllll}
\hline Correction & Validity range & Predictors & $R^{2}$ & P-value \\
\hline$c_{l i n}^{*}$ & $R \leq 1$ & $c_{A v} ; \bar{u}$ & 0.62 & 0.01 \\
$c_{l i n}^{*}$ & $R>1$ & $c_{A v} ; \bar{u}$ & 0.78 & $1.04 \cdot 10^{-10}$ \\
$c_{l i n}^{* *}$ & Entire data range & $c_{\text {lin }}^{*}$ & 0.85 & $2.2 \cdot 10^{-16}$ \\
& \multicolumn{4}{c}{ Coefficients } \\
$c_{l i n}^{*}$ & $R \leq 1$ & $0.91\left(\alpha_{1}\right)$ & $225.93\left(\alpha_{2}\right)$ \\
$c_{l i n}^{*}$ & $R>1$ & $36.09\left(\alpha_{3}\right)$ & $176.02\left(\alpha_{4}\right)$ \\
$c_{\text {lin }}^{* *}$ & Entire data range & $1.17\left(\beta_{1}\right)$ & \\
\hline
\end{tabular}

of $\mathrm{A}_{\mathrm{v}}$ samplers, assuming a birch pollen diameter $d=22 \mu \mathrm{m}$ (Birks 1968) and a density $\gamma=800 \mathrm{~kg} \mathrm{~m}^{-3}$. The measured aspiration efficiency of the vertical samplers was determined with $E_{A v}=c_{A v} / c_{B}$. Note that the aspiration of the B sampler underlies the same inertial effects discussed above. Its bulky body presents a considerable obstacle to the freestream, which distorts the freestream as it approaches the sampler. Since the B sampler was designed to rather register inhalable aerosols, its readings do not exactly represent the atmospheric concentration. The 'true' aspiration efficiency of the B sampler was determined by Ogden et al. (1974) using Ambrosia pollen $(20 \mu \mathrm{m})$. A mathematical equation for the empirical data was provided by Frenz (1999). Figure 6 shows that the B sampler aspiration declines to $60 \%$ at $U=5 \mathrm{~ms}^{-1}$. With increasing wind velocity, the aspiration efficiency exceeds $100 \%$. Hence, measurements aiming at the atmospheric aerosol concentration need to be corrected with respect to wind velocity. The physically based aspiration model, however, was derived also from studies on inhalability by the human head. Therefore, comparison of the model to the uncorrected B sampler seems reasonable.

Figure 7 shows that the physically based model dramatically overestimates the aspiration efficiency of the $A_{v}$

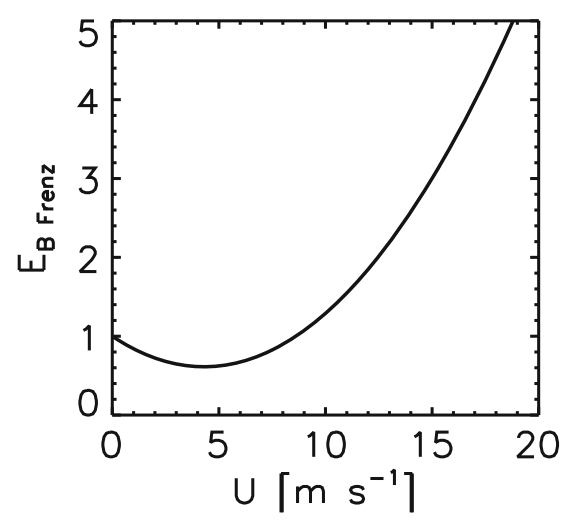

Fig. 6 Aspiration efficiency of the Burkard sampler as a function of the wind velocity $U$, using the mathematical equation provided by Frenz (1999) based on the empirical data of Ogden et al. (1974)

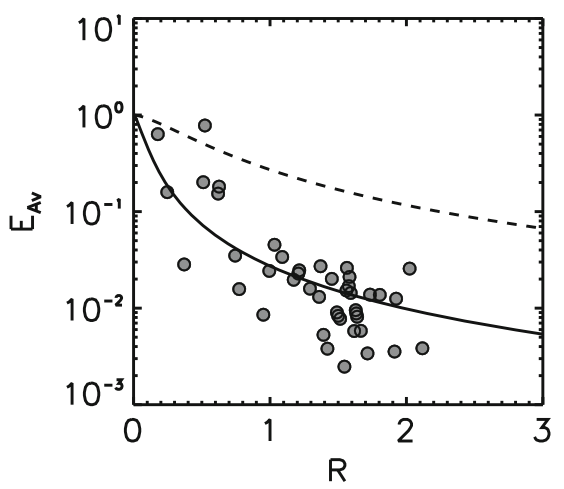

Fig. 7 Measured aspiration efficiency $E_{A v}=c_{A v} / c_{B}$ plotted as a function of $R=U / U_{\mathrm{s}}$, where $U$ is the freestream velocity and $U_{\mathrm{s}}$ is the sampling velocity of the Air-O-Cell sampler $\left(1.84 \mathrm{~ms}^{-1}\right)$. Solid curve Modeled aspiration efficiency $E_{90}$ using $G_{90}=2.21$ and $g_{1}=-1$, dashed curve modeled (Eq. 9) using $G_{90}=29.35$ and $g_{1}=-0.5$. Note that the $y$ axis is logarithmic

sampler, when the empirical value $G_{l}=2.21$ determined from human head studies (Tsai and Vincent 1993), is used. The best results (i.e., the lowest bias) for the $\mathrm{A}_{\mathrm{v}}$ data yields $G_{90}=29.35$ with $g_{1}=-0.5$. Thus the parameter $G_{90}$ needs substantial modification for the vertical A sampler, since the greater bluntness of the sampler body must be taken into account. It seems that the exact value of $G_{90}$ is a strong function of the very sampler design. To what extent this value can be considered characteristic for an Air-O-Cell sampler depends on the dimension of the pump housing. Thus it would be helpful to determine the dependence of $G_{90}$ on different sampler designs. The model was used with $G_{90}=29.35$ to correct the vertically measured data through:

$c_{p h y s}^{*}=\frac{1}{E_{90}} c_{A v}$,

where $c_{\text {phys }}^{*}$ denotes the corrected $\mathrm{A}_{\mathrm{v}}$ pollen concentration. Note that the data are corrected only when the deviation from B samplers is larger than the rmse of the B samplers. The correlation of the corrected data $\left(R^{2}=0.39\right)$ is considerably less than that of Tsai and Vincent (1993) for their aerosols of aerodynamic diameters up to $60 \mu \mathrm{m}$. The model generally underestimates the measurements when $R \leq 1$, and generally overestimates when $R>1$. The relative bias and relative rmse between $c_{p h y s}^{*}$ and $c_{\mathrm{B}}$ is $0 \%$ and $89 \%$, respectively. Figure 8 a shows that the corrected data as a function of $c_{B}$ are widely scattered. A significant correlation can be found only due to a satisfying agreement for pollen concentrations $c>1 \times 10^{3}$ pollen $\mathrm{m}^{-3}$. Since no pollen counts are considered in Eq. 9, the physically based correction can not take into account errors that are induced by effects other than the aspiration efficiency, e.g., the error due to the extrapolation of A sampler data, as discussed earlier. The errors of the uncorrected and corrected data are summarized in Table 3. 
Fig. 8 a Corrected $1-\mathrm{h} \mathrm{A}_{\mathrm{V}}$ data $\left(c_{\text {phys }}^{*}\right)$ using the physically based model, as function of $c_{B}$. b Corrected 1-hour $\mathrm{A}_{\mathrm{v}}$ data $\left(c_{l i n}^{* *}\right)$ using the linear model, as function of $c_{B}$. Note that the plots are double-logarithmic
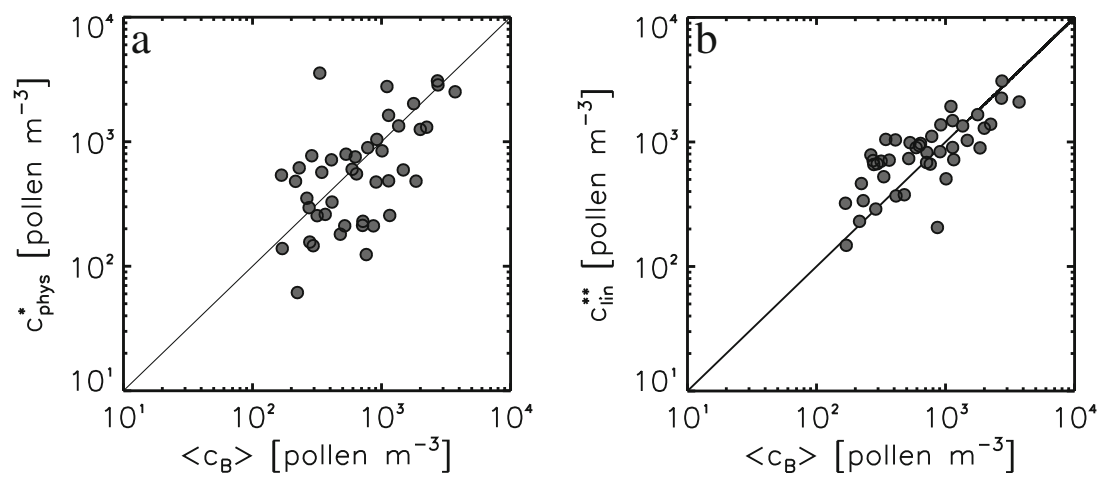

Performance of the linear correction model

The performance of the linear correction was tested against B sampler measurements. The linear model was used to correct the vertically measured data. Note that the data are corrected only when the deviation from B samplers is larger than the rmse of the B samplers. The correlation of the corrected data is $R^{2}=0.63$ and the relative rmse is $57 \%$. Although the error is still relatively large, it is not substantially larger than the precision error of the B sampler (Table 1). The relative bias of $3 \%$ indicates a slight overestimation of the corrected $c_{\text {lin }}^{* *}$. Figure $8 \mathrm{~b}$ shows that the scatter of the corrected data $c_{\text {lin }}^{* *}$ as function of $c_{B}$ is considerably less than in the case of the physical model. The linear correction takes into account the measured pollen concentration. Therefore, errors resulting from, e.g., the extrapolation of A sampler data, are taken into account to a certain extent. The errors of the uncorrected and corrected data are summarized in Table 3 .

Table 3 Summary of relative bias (mean deviation normalized by the corresponding mean pollen concentration $\langle\bar{c}\rangle$ shown in Table 1) and relative rmse (normalized by the corresponding mean pollen concentration $\langle\bar{c}\rangle$ shown in Table 1) between uncorrected and corrected $\mathrm{A}_{\mathrm{V}}$ and $\mathrm{B}$ samplers (reference) using the physically based and the linear model, respectively. $c_{A v}$ denotes uncorrected measurements of the vertically oriented $\mathrm{A}_{\mathrm{v}}$ sampler, $c_{A h}$ denotes measurements of the horizontally oriented $\mathrm{A}_{\mathrm{h}}$ sampler, $c_{B}$ denotes measurements of the $\mathrm{B}$ sampler, $c_{\text {lin }}^{* *}$ denotes the corrected $c_{A v}$ data using the linear model (Eq. 12) and $c_{p h y s}^{*}$ denotes the corrected $c_{A v}$ data using the physically based model (Eq. 9). The values of the relative bias and relative rmse are given in percent

\begin{tabular}{llllllll}
\hline Data & \multicolumn{2}{l}{$c_{A h}$ (Reference) } & & & \multicolumn{2}{c}{$c_{B}$ (Reference) } \\
\cline { 2 - 3 } \cline { 6 - 7 } & $\begin{array}{l}\text { Relative } \\
\text { bias }\end{array}$ & $\begin{array}{l}\text { Relative } \\
\text { rmse }\end{array}$ & $R^{2}$ & & $\begin{array}{l}\text { Relative } \\
\text { bias }\end{array}$ & $\begin{array}{l}\text { Relative } \\
\text { rmse }\end{array}$ & $R^{2}$ \\
\hline$c_{A v}$ & -105 & 154 & 0.00 & -104 & 140 & 0.00 \\
$c_{A h}$ & - & - & - & -20 & 50 & 0.76 \\
$c_{\text {lin }}^{*}$ & 9 & 74 & 0.60 & - & - & - \\
$c_{\text {lin }}^{* *}$ & - & - & - & 3 & 57 & 0.63 \\
$c_{\text {phys }}^{*}$ & - & - & - & 0 & 89 & 0.39 \\
\hline
\end{tabular}

\section{Conclusions}

The present work quantified in a field experiment the uncertainty of vertically oriented blunt aerosol sampling systems using Air-O-Cell cassettes. The investigation focussed on the decrease of vertical pollen aspiration (hazel, sweet chestnut and birch) due to inertial effects on aerosol motion in moving air. The aspiration efficiency of the vertical samplers was determined with wind-oriented reference samplers (Hirst-type Burkard sampler, horizontal Air-O-Cell sampler). The decrease of aspiration efficiency of the vertically oriented sampler resulted in a substantial underestimation of the pollen count relative to horizontal measurements $(-104 \%$ relative bias). This underlines the importance of applying a correction when the sampler inlet is facing upwards, as is quite common in, e.g., aeroallergenic research. Wind-oriented mounting of the same sampler type yields an considerably smaller underestimation relative to horizontal measurements ( $-20 \%$ relative bias). A physically based semi-empirical correction model was verified with birch pollen measurements for the purpose of the vertically oriented Air-O-Cell sampler. Additionally, a linear model was developed on the basis of pollen counts and wind velocity measurements. Both models were capable of notably reducing the error induced by vertical sampling. In comparison to the physical model, the linear correction yielded a better agreement to the reference. The linear model, however, is valid only under the very conditions of the experimental site and for the specific sampler design. The physically based model, in contrast, can be transferred to each upwards-facing blunt sampler type. It should be noted, however, that the characteristic bluntness of a sampler might make a modification of the semi-empirical model necessary in order to provide a robust correction.

The precision error of the wind-oriented Air-O-Cell sampler is rather large ( $26 \%$ of the mean concentration), yet is smaller than the precision error found for the Burkard sampler (38\%). The Air-O-Cell cassette itself, therefore, can be considered a good low-priced alternative to, e.g., the widely used Burkard sampler. Attention should focus on the pump housing that is attached to the sampling cassette, however, since it may strongly affect the aspiration efficiency. 
The results of the sampler intercomparison clearly point out that aspiration of aerosols (i.e., heavy aerosols such as pollen) should always be wind-oriented. In the case of blunt samplers and non-isokinetic sampling, the sampling error is a function of wind velocity, also in the case of a windoriented inlet. Thus under field conditions, a physically based correction of pollen counts is indispensable.

Acknowledgments Our great appreciation goes to the European Cooperation in Science and Technology (COST) Action ES0603. Financial support for this project by the Swiss State Secretariat for Education and Research (SBF), grant C07.0111, and the Freiwillige Akademische Gesellschaft Basel, is gratefully acknowledged. Many thanks go to MeteoSwiss and the MCR Lab, University of Basel. Their contribution to the field campaigns and the pollen analysis is highly appreciated. The comments of an anonymous reviewer to a first draft of the present paper led to a substantial improvement of the results from this study and are greatly acknowledged.

\section{References}

Aizenberg V, Reponen T, Grinshpun S, Willeke K (2000) Performance of Air-O-Cell, Burkard and Button Samplers for total enumeration of airborne spores. Am Ind Hyg Assoc J 61:855-864

Armbruster L, Breuer H (1982) Investigations into defining inhalable dust. Pergamon, Oxford, pp 3-19

Badzioch S (1959) Collection of gas-borne dust particles by means of an aspirated sampling nozzle. Br J Appl Phys 10:26-32

Belyaev S, Levin L (1974) Techniques for collection of representative aerosol samples. J Aerosol Sci 5:325-338

Birks H (1968) The identification of betula nana pollen. New Phytol 67:309-314

Bucher E, Kofler V, Vorwohl G, Zieger E (2004) Das Pollenbild der Südtiroler Honige. Landesagentur für Umwelt und Arbeitsschutz, Biologisches Labor

Cariñanos P, Emberlin J, Galán C, Domínguez-Vilches E (2000) Comparison of two pollen counting methods of slides from a hirst type volumetric trap. Aero 16:339-346

Comtois P, Alcazar P, Neron D (1999) Pollen counts statistics and its relevance to precision. Aerobiologia 15:19-28

Dunnett S, Wen X, Zaripov S, Galeev R, Vanunina M (2006) A numerical study of calm air sampling with a blunt sampler. Aerosol Sci Technol 40:490-502

Durham M, Lundgren D (1980) Evaluation of aerosol aspiration efficiency as function of stokes number, velocity ratio and nozzle angle. J Aerosol Sci 11:179-188
Frenz D (1999) Comparing pollen and spore counts collected with the Rotorod sampler and Burkard spore trap. Ann Allergy Asthma Immunol 83:341-347

Gottardini E, Cristofolini F, Cristofori A, Vannini A, Ferretti M (2009) Sampling bias and sampling errors in pollen counting in aerobiological monitoring in Italy. J Environ Monitor 11:751-755

Grinshpun S, Lipatov G (1990) Sampling errors in cylindrical nozzles. Aerosol Sci Technol 12:716-740

Grinshpun S, Chang CW, Nevalainen A, Willeke K (1994) Inlet characteristics of bioaerosol samplers. J Aerosol Sci 25 (8):1503-1522

Hangal S, Willeke K (1990a) Aspiration efficiency: unified model for all forward sampling angles. Environ Sci Technol 24:688-691

Hangal S, Willeke K (1990b) Overall efficiency of tubular inlets sampling at 0-90 degrees from horizontal aerosol flows. Atmos Environ 24A:2379-2386

Hirst J (1952) An automatic volumetric spore trap. Ann Appl Biol 39:257-265

Levetin E (2004) Methods for aeroallergen sampling. Curr Allergy Asthma Rep 4:376-383

Lipatov G, Grinshpun S, Semenyuk T, Sutugin A (1988) Secondary aspiration of aerosol particles into thin-walled nozzles facing the wind. Atmos Environ 22:1721-1727

Lundgren D, Durham M, Mason K (1978) Sampling of tangential flow streams. Am Ind Hyg Assoc J 39:640644

Mandrioli P, Comtois P, Levizzani V (1998) Methods in aerobiology. Pitagora Editrice, Bologna, Italy

Michel D, Gehrig R, Rotach M, Vogt R (2010) Micropoem: experimental investigation of birch pollen emission. In: 19th Symposium on Boundary Layer and Turbulence, 2-8 August, Keystone, Colorado

Ogden T, Birkett J (1977) The human head as a dust sampler. Pergamon, Oxford, pp 93-105

Ogden E, Raynor G, Hayes J, Lewis D, Haines J (1974) Manual for sampling airborne pollen. Hafner, New York

Paik S, Vincent J (2002) Aspiration efficiency for thin-walled nozzles facing the wind and for very high velocity ratios. J Aerosol Sci $33: 705720$

Tsai P, Vincent J (1993) Impaction model for the aspiration efficiencies of aerosol samplers at large angles with respect to the wind. J Aerosol Sci 24:919-928

Vincent J (1989) Aerosol sampling: science and practice. Wiley, Chichester

Vincent J (2007) Aerosol sampling: science, standards, instrumentation and applications. Wiley, Chichester

Vincent J, Stevens D, Mark D, Marshall M, Smith T (1986) On the spiration characteristics of large-diameter, thin-walled aerosol sampling probes at yaw orientations with respect to the wind. $\mathrm{J}$ Aerosol Sci 17:211-224

Wiener R, Okazaki K, Willeke (1988) Influence of turbulence on aerosol sampling efficiency. Atmos Environ 22:917-928 